\section{Discovery of a possible hybrid of the Critically Endangered Forest Owlet Athene blewitti and Spotted Owlet Athene brama (Aves: Strigiformes) from northern Maharashtra, India}

\author{
Satish A. Pande ${ }^{1}$, Amit P. Pawashe ${ }^{1}$, Raju \\ Kasambe ${ }^{2}$ \& Reuven Yosef ${ }^{2,3}$
}

${ }^{1}$ Ela Foundation, C-9 Bhosale Park, Sahakar Nagar-2, Pune, Maharashtra 411009, India

${ }^{2}$ Sevadal Mahila Mahavidyalaya, Sakkardara chowk, Umrer Road, Nagpur, Maharashtra 440009, India

${ }^{3}$ International Birding \& Research Centre in Eilat, P.O. Box 774, Eilat 88000, Israel

Email: ${ }^{3}$ ryosef@eilatcity.co.il (corresponding author)

Owls (Strigiformes) are considered to have the lowest hybridization rate among birds (Mikkola 2003). It is believed that inherent isolation mechanisms in the owls are relatively effective in explaining the low frequency of hybridization between sympatric owl species (Mikkola 1983, 2003).

The Forest Owlet Athene blewitti was recently rediscovered after 113 years in western and central India (King \& Rasmussen 1998), specifically in the states of Madhya Pradesh and Maharashtra (Rasmussen

Date of publication (online): 26 April 2011

Date of publication (print): 26 April 2011

ISSN 0974-7907 (online) | 0974-7893 (print)

Editor: Boris P. Nikolov

Manuscript details:

Ms \# 02553

Received 28 August 2010

Final received 06 December 2010

Finally accepted 16 January 2011

Citation: Pande, S.A., A.P. Pawashe, R. Kasambe \& R. Yosef (2011) Discovery of a possible hybrid of the Critically Endangered Forest Owlet Athene blewitti and Spotted Owlet Athene brama (Aves: Strigiformes) from northern Maharashtra, India. Journal of Threatened Taxa 3(4): 1727-1730.

Copyright: (C) Satish A. Pande, Amit P. Pawashe, Raju Kasambe \& Reuven Yosef 2011. Creative Commons Attribution 3.0 Unported License. JoTT allows unrestricted use of this article in any medium for non-profit purposes, reproduction and distribution by providing adequate credit to the authors and the source of publication.

Acknowledgements: We thank Nathaniel J. Moses, Satish Ranade, Keda Pawagi, and Tzadok Tzemach for their help in the field; and the officials and staff of the Indian Forest Department in Nagpur, Pune, and MTR for their support. Special mention is due to Mr R. Choudhary, CCF, MTR, Amravati, and Mr Prakash Thosare, CCF Pune, for their help in making our stay in the remote forest areas possible. Optics donated by Swarovski Optics facilitated our fieldwork. We greatly thank our anonymous donor who underwrote the expenses of the expedition.

\section{OPEN ACCESS | FREE DOWNLOAD (C) (i) (4)}

\& Collar 1998; Pande et al. 2003; Rithe 2003). Little is known about this endemic species, which has a very limited distribution in central India, and to date limited studies have been undertaken on their ecology and behavior (e.g., Rasmussen \& Ishtiaq 1999; Ishtiaq et al. 2002).

The species is considered to be Critically Endangered and at an extremely high risk of extinction (Collar et al. 1994; del Hoyo et al. 1999). The species is listed under Schedule I of the Indian Wildlife (Protection) Act and hence we are unable to collect any kind of samples (feces, pellets, discarded feathers, etc.) or to capture and sample any of the birds for molecular studies. Hence, our study is based on visual observations (which include photographic and video 1 $\& 2$ ) of the individuals reported here.

\section{Study Area and Methods}

To further enhance our understanding of the species' habitat requirements, the Ela Foundation (ELA) and the International Birding and Research Centre in Eilat (IBRCE) arranged an expedition to the Melghat Tiger Reserve (MTR) in early February 2004. In the framework of this project, we undertook transects in MTR and mapped all known and newly discovered territories of Forest and Spotted Owlets.

Because of the fact that the species is Critically Endangered, our activities were constantly monitored by a forest ranger who also prevented us from collecting any feather, feces or pellet samples of the owls.

\section{Results and Discussion}

During our study we became well acquainted with the different color morphs and behavioral idiosyncrasies of the two sympatric species. This enabled us to discern that a particular territory bordering on Forest and Spotted Owlet (A. b. indica) territories had owls with intermediate plumages, vocalizations, and behaviors. The family that defended the territory consisted of two adults and one recently fledged owlet. Since all our studies were photographed and videotaped, we were able to compare the two Athene species with this family, and we concluded that it was a fertile hybrid of the two species. The larger of the two adults was assumed to be the female and was verified in subsequent days when copulation was 



Figure 1. Field sketches to stress characteristics of the Spotted Owlet (Athene brama); (a) Forest Owlet (A. blewitti); (b) hybrid (frontal view); (c) and lateral view (d). Photographs are inset to show field observations.
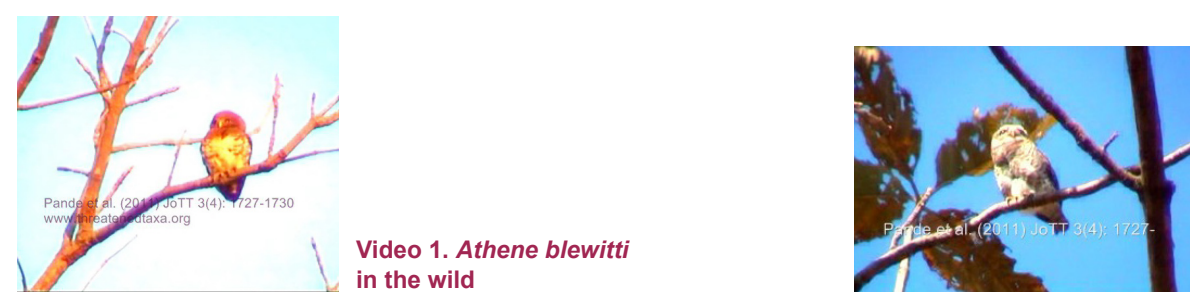

Video 2. Possible hybrid of Athene blewitti and Athene brama in the wild 




Fig. 2. Comparison of vocalization duration (in seconds) of the Spotted (Athene brama) and Forest Owlet (A. blewitti) at Melghat Tiger Reserve. The numbers of each bar denote sample size (N).

observed. All data were obtained from still and video photographic evidence collated by the authors, and are based on visual observations in the field (Fig. 1).

The Spotted Owlet (Fig. 1a) has a heavily spotted crown, a darkish facial disc bordered by white around its sides; and curved white eyebrows (del Hoyo et al. 1999; this study). Its ear coverts are white. The dorsal side is gray-brown with scattered white spots. Its tail is short and has a conspicuous white-tip; the white tail bars are narrow. The breast is creamy white with short gray to brownish bars, and has a broad white gorget. Tail wagging is vertical (up and down) only. The duration of its call is $9 \mathrm{~s}( \pm 1.3, \mathrm{~N}=11)$ and it vocalizes all night (Fig. 2).

In contrast, the Forest Owlet (Fig. 1b) has a mostly white facial disc with fine light brown to dark-brown barring (del Hoyo et al. 1999; this study). Its white facial disc is almost invisible in the field and its face appears brown. The head is very sparsely spotted and in many individuals it appears unspotted, as are the mantle and the back. Interestingly, it has an obsolete hind-collar, thin white eyebrows, and its flight feathers and rectrices have broad white bars. The tail is short and has a broad, white terminal bar that is visible below the wings when the owl is perched. The Forest Owlet's ventral region is mostly white with a dark chocolate brown bar across the neck and a broad lateral band on the top third of its chest. The lateral lower breast and upper flanks are broadly barred and the central ventral region is white, in the shape of an inverted "U". To date, juvenile owlets have not been fully characterized. The juvenile owlet that we observed had a buff-colored central breast; in adults the lower abdomen is white. Tail wagging is lateral (sideways) only. The duration of its call is ca. 3s ( \pm $0.8, \mathrm{~N}=73$; Fig. 2) and it vocalizes only during the day. When it vocalizes, its head is extended and the thin white gular band is visible.

In addition, we also discovered individuals which displayed a combination of the markings of the two species and whose vocalizations were intermediate. This has led us to believe that these are most probably hybrids of the Forest and Spotted owlets that coexist in the forests.

As expected, the hybrid individuals displayed characteristics that were intermediate to the two species described above (Fig. 1c). Two of the three birds had a few faint white spots on the head, a thin white-eye ring and eyebrows, but no white on the ear coverts. A thin white throat band was visible when it was resting and also when the bird stretched its neck or was engaged in vocalizing. The upper part of the chest was dark brown but fainter than in the Forest Owlet and the lower two-thirds of the chest had streaks shaped like inverted arrowheads on a white background. Notably, barring on the flanks was absent. The portion of the tail that protruded beyond the wings had a sub-terminal dark band and a white terminal band. Appreciable spotting on the wings was noted when the owl was viewed from the side or behind (Fig. 1d). Its dorsal color was brown and paler than that of the Forest Owlet, and was characterized by a grayish tinge and significant white spotting. The primaries were black. Surprisingly, the characteristic that alerted us to the possibility of these being hybrids was the tail wagging, which was lateral and horizontal. The duration of its call was ca. $6 \mathrm{~s}$ ( \pm $0.9, \mathrm{~N}=34)$ and it vocalized during the day (0500$1100 \mathrm{hr}$ ) and from after noon until early night (1600$1900 \mathrm{hr}$ ). These findings are similar to that reported for hybrids between the Barred (Strix occidentalis) and Spotted Owls (S. varia) that give calls that are intermediate between the typical calls of the two species (del Hoyo et al. 1999).

The differences described above also puzzled the local indigenous guide, from the Korku Adivasi tribe, who has been well-acquainted with the owls of the region for several decades, thus suggesting that this was a unique or infrequent situation. Also, RK showed a photograph of a similar individual in 2002, photographed in the same area, but unfortunately he was unable to identify it conclusively. This suggests 
that the hybrids may have a much wider distribution that could equal or surpass the very limited one of the Forest Owlet.

Hybridization is comparatively well known in owls (del Hoyo et al. 1999). We assume that several demographic factors have influenced the populations involved and allowed them to cross the species-limit barrier and for hybridization to occur. The logic that the Forest Owlet is (i) limited geographically to the Satpura Range, (ii) attitudinally to the higher, forested parts, (iii) limited to habitats in the proximity of humans and resulting clearings that facilitate foraging (Yosef et al. submitted), and (iv) limited demographically to low population levels wherein (v) neighbouring territories are located far apart, leads us to assume hybridization with the far more common Spotted Owlet. It is possible that dispersing individuals that do not find conspecifics set up territories and mate with the abundant Spotted Owlets.

Our observations strongly suggest that the hybrids are fertile; the female was subsequently observed (and filmed) while engaged in extra-pair copulation (EPC) with the neighboring male Forest Owlet. The biological implications of our discovery regarding conservation and our detailed description of the hybrids of the Critically Endangered Forest Owlet, thus confirming their existence, is of great interest in light of the fact that hybridization is rare in owls, but its consequences can be far-reaching and grave (Haig et al. 2004). Many a wild species have been known to loose their identity and legal protection, owing to genetic contamination, making their continued conservation a philosophically problematic question that could result in chaos (Sutherland 2000).

\section{REFERENCES}

Collar, N.J., M.J. Crosby \& A.J. Stattersfield (1994). Birds to Watch 2: The World List of Threatened Birds.Birdlife Conserv. Series no. 2. Birdlife International, Cambridge, 407pp.

Del Hoyo J., A. Elliott \& J. Sargatal (eds.)(1999). Handbook of the Birds of the World. Vol. 5. Barn Owls to hummingbirds. Lynx Edicions, Barcelona, 759pp.

Haig S.M., T.D. Mullins, E.D. Forsman, P.W. Trail \& L. Wennerberg (2004). Genetic identification of Spotted Owls, Barred Owls and their hybrids: legal implications of hybrid identity. Conservation Biology 18: 1347-1357.

Ishtiaq, F., A.R. Rahmani \& P.C. Rasmussen (2002). Ecology and behaviour of the Forest Owlet (Athene blewitti), pp. 8088. In: Newton, I., R. Kavanagh, J. Olsen \& I. Taylor (eds.). Ecology and Conservation of Owls. CSIRO Publishing, Australia, 363pp.

King, B.F. \& P.C. Rasmussen (1998). The rediscovery of the Forest Owlet Athene (Heteroglaux) blewitti. Forktail 14: 51-53.

Mikkola, H. (1983). Owls of Europe. T \& A.D. Poyser, U.K., 397pp.

Mikkola, H. (2003). Strangers in the dark: hybridization between owl species, pp. 82-87. In: Duncan, J.R. (ed.). Owls of the World. Key Porter books, Ltd., Toronto, Canada, 319pp.

Pande, S., S. Tambe, C.M. Francis \& N. Sant (2003). Birds of Western Ghats, Konkan and Malabar (including birds of Goa). Oxford University Press, Pune, India, 375pp.

Rasmussen, P.C. \& F. Ishtiaq (1999). Vocalizations and behaviour of the Forest Owlet Athene (Heteroglaux) blewitti. Forktail 15: 61-65.

Rithe, K. (2003). Saving the Forest Owlet. Sanctuary Asia February: 30-33.

Sutherland, W.J. (2000). The Conservation Handbook: Research, Management and Policy. Blackwell Science, Ltd., Oxford, 278pp.

Yosef, R., S.A. Pande \& R. Kasambe (submitted). Anthropogenic activity aids habitat selection and survival of the Critically Endangered Forest Owlet (Athene blewitti). Journal of Threatened Taxa. 